\title{
Hadamard and Jensen inequalities for s-convex fuzzy processes
}

\author{
R. Osuna-Gómez ${ }^{\mathrm{a}}$, M.D. Jiménez-Gamero a, \\ Y. Chalco-Cano ${ }^{\mathrm{b}, 1}$ and M.A. Rojas-Medar ${ }^{\mathrm{b}, 2}$ \\ ${ }^{a}$ Departamento de Estadística e Investigación Operativa Facultad de Matemáticas, \\ Universidad de Sevilla, 41012 Sevilla, Spain \\ ${ }^{\mathrm{b}}$ IMECC-UNICAMP, CP 6065, 13083-970, Campinas-SP, Brazil
}

\begin{abstract}
We give some inequalities of Hadamard and Jensen type for s-convex fuzzy processes. We also give some applications.
\end{abstract}

Key words: fuzzy sets, s-convex process, Hadamard and Jensen inequalities

\section{Introduction}

In [1] the s-convex fuzzy processes were defined and some properties were studied. In this work, we define the s-concave fuzzy processes and we also give some useful inequalities for both, the s-convex and s-concave fuzzy processes.

The paper has the following structure. In Section 2, we fix some basic notation and terminology. In Section 3, we define the s-concave fuzzy process and we give some properties. In Section 4, we establish the Hadamard inequality. In Section 5, we give a generalization of the Jensen inequality.

^ Partially financed by the Ministerio de Ciencia y Tecnología, Spain, Grant BFM2003-06579

Email addresses: rafaela@us.es (R. Osuna-Gómez), dolores@us.es (M.D. JiménezGamero), katary@ime.unicamp.br (Y. Chalco-Cano), marko@ime.unicamp.br (M.A. Rojas-Medar).

1 Y. Chalco-Cano is supported by Fapesp-Brasil, grant No 00/00055-0.

2 M.A. Rojas-Medar is partially supported by CNPq-Braszil, grant 300116/93(RN). 


\section{Preliminaries}

Let $\mathbb{R}^{n}$ denote the $n$-dimensional Euclidean space and let $C \subseteq \mathbb{R}^{n}$ denote a convex set. Let $s \in(0,1]$ and let $f: C \subseteq \mathbb{R}^{n} \rightarrow \mathbb{R}$ be a function such that for all $a \in[0,1]$ and for all $x, y \in C$, the following inequality holds

$$
f\{a x+(1-a) y\} \leq a^{s} f(x)+(1-a)^{s} f(y)
$$

These functions are called s-convex and they have been introduced by Breckner [2], where it is also possible to find examples of s-convex functions (see also $[3])$.

Let $P\left(\mathbb{R}^{n}\right)$ denote the set of all nonempty subsets of $\mathbb{R}^{n}$. In [4], Breckner generalized the notion of s-convexity for a set-valued mapping $F: C \subseteq \mathbb{R}^{m} \rightarrow$ $P\left(\mathbb{R}^{n}\right) . F$ is said to be a s-convex function on $C$ if the following relation is verified

$$
(1-a)^{s} F(x)+a^{s} F(y) \subseteq F\{(1-a) x+a y\}
$$

for all $a \in[0,1]$ and all $x, y \in \mathbb{R}^{m}$.

We denote by $\mathcal{K}\left(\mathbb{R}^{m}\right)$ the subset of $P\left(\mathbb{R}^{m}\right)$ whose elements are compact and nonempty and by $\mathcal{K}_{c}\left(\mathbb{R}^{m}\right)$ the subset of $\mathcal{K}\left(\mathbb{R}^{m}\right)$ whose elements are convex. If $A \in \mathcal{K}\left(\mathbb{R}^{m}\right)$, then the support function $\sigma(A, \cdot): \mathbb{R}^{m} \rightarrow \mathbb{R}$ is defined as

$$
\sigma(A, \psi)=\sup _{a \in A}<\psi, a>, \forall \psi \in \mathbb{R}^{m}
$$

It is important to remark that if $A, B \in \mathcal{K}_{c}\left(\mathbb{R}^{m}\right)$, then, as a direct consequence of the separation Hahn-Banach theorem, we obtain that $\sigma(A, \cdot)=\sigma(B, \cdot) \Leftrightarrow$ $A=B$.

A fuzzy subset of $\mathbb{R}^{n}$ is a function $u: \mathbb{R}^{n} \rightarrow[0,1]$. Let $\mathcal{F}\left(\mathbb{R}^{n}\right)$ denote the set of all fuzzy sets on $\mathbb{R}^{n}$. We define the addition and the scalar multiplication on $\mathcal{F}\left(\mathbb{R}^{n}\right)$ by the usual extension principle as follows:

$$
(u+v)(y)=\sup _{y_{1}, y_{2}: y_{1}+y_{2}=y} \min \left\{u\left(y_{1}\right), v\left(y_{2}\right)\right\}
$$

and

$$
(\lambda u)(y)= \begin{cases}u\left(\frac{y}{\lambda}\right) & \text { if } \lambda \neq 0, \\ \chi_{\{0\}}(y) & \text { if } \lambda=0,\end{cases}
$$

where for any subset $A \subseteq \mathbb{R}^{n}, \chi_{A}$ denotes the characteristic function of $A$. 
We can define a partial order $\subseteq$ on $\mathcal{F}\left(\mathbb{R}^{n}\right)$ by setting

$$
u \subseteq v \Leftrightarrow u(y) \leq v(y), \quad \forall y \in \mathbb{R}^{n}
$$

Let $u \in \mathcal{F}\left(\mathbb{R}^{n}\right)$. For $0<\alpha \leq 1$, we denote by $[u]^{\alpha}=\left\{y \in \mathbb{R}^{n} \quad / \quad u(y) \geq \alpha\right\}$ the $\alpha$-level set of $u$. $[u]^{0}=\operatorname{supp}(u)=\overline{\left\{y \in \mathbb{R}^{n} \quad / \quad u(y)>0\right\}}$ is called the support of $u$.

A fuzzy set $u$ is called convex if (see [5])

$$
u\left\{\lambda y_{1}+(1-\lambda) y_{2}\right\} \geq \min \left\{u\left(y_{1}\right), u\left(y_{2}\right)\right\}
$$

for all $y_{1}, y_{2} \in \operatorname{supp}(u)$ and $\lambda \in(0,1)$. If $u \in \mathcal{F}\left(\mathbb{R}^{n}\right)$ is convex, then $[u]^{\alpha}$ is convex for all $\alpha \in[0,1]$.

A fuzzy set $u: \mathbb{R}^{n} \rightarrow[0,1]$ is said to be a fuzzy compact set if $[u]^{\alpha}$ is compact for all $\alpha \in[0,1]$. We denote by $\mathcal{F}_{K}\left(\mathbb{R}^{n}\right)\left(\mathcal{F}_{C}\left(\mathbb{R}^{n}\right)\right)$ the space of all fuzzy compact (compact convex) sets. Given $u, v \in \mathcal{F}_{K}\left(\mathbb{R}^{n}\right)$, it is verified that

(a) $u \subseteq v \Leftrightarrow[u]^{\alpha} \subseteq[v]^{\alpha}, \quad \forall \alpha \in[0,1]$

(b) $[\lambda u]^{\alpha}=\lambda[u]^{\alpha}, \quad \forall \lambda \in \mathbb{R}, \forall \alpha \in[0,1]$,

(c) $[u+v]^{\alpha}=[u]^{\alpha}+[v]^{\alpha}, \quad \forall \alpha \in[0,1]$.

Any application $F: \mathbb{R}^{m} \rightarrow \mathcal{F}\left(\mathbb{R}^{n}\right)$ is called a fuzzy process. For each $\alpha \in[0,1]$ we define the set-valued mapping $F_{\alpha}: \mathbb{R}^{m} \rightarrow P\left(\mathbb{R}^{n}\right)$ by

$$
F_{\alpha}(x)=[F(x)]^{\alpha} .
$$

For any $u \in \mathcal{F}_{C}\left(\mathbb{R}^{n}\right)$ the support function of $u, S(u,(\cdot, \cdot)):[0,1] \times \mathbb{S}^{m} \rightarrow \mathbb{R}$, where $\mathbb{S}^{m}=\left\{\psi \in \mathbb{R}^{m} /\|\psi\| \leq 1\right\}$, is defined as

$$
S(u,(\alpha, \psi))=\sigma\left([u]^{\alpha}, \psi\right) .
$$

For details about support functions see for example [6].

A fuzzy process $F: \mathbb{R}^{m} \rightarrow \mathcal{F}\left(\mathbb{R}^{n}\right)$ is called convex if it satisfies the following relation

$$
F\left\{(1-a) x_{1}+a x_{2}\right\}(y) \geq \sup _{y_{1}, y_{2}:(1-a) y_{1}+a y_{2}=y} \min \left\{F\left(x_{1}\right)\left(y_{1}\right), F\left(x_{2}\right)\left(y_{2}\right)\right\}
$$

for all $x_{1}, x_{2} \in \mathbb{R}^{m}, a \in(0,1)$ and $y \in \mathbb{R}^{n}$. This notion of convex fuzzy processes was recently introduced in [7]. This definition extend the Matloka definition given in [8]. 


\section{S-convex fuzzy processes}

In [1] the authors introduced the definition of s-convex fuzzy processes as follows.

Definition 1 Let $s \in(0,1]$. A fuzzy process $F: C \subseteq \mathbb{R}^{m} \rightarrow \mathcal{F}\left(\mathbb{R}^{n}\right)$ is said to be a s-convex fuzzy process on $C$, if for all $a \in(0,1)$ and for all $x, y \in C$ it satisfies the condition

$$
(1-a)^{s} F(x)+a^{s} F(y) \subseteq F\{(1-a) x+a y\}
$$

This definition is a generalization of the notion of s-convexity for a set-valued mapping given in (2), since if $\Gamma: C \subseteq \mathbb{R}^{m} \rightarrow P\left(\mathbb{R}^{n}\right)$ is a set-valued mapping, then by putting $F(x)=\chi_{\Gamma(x)}$, we see that Definition 1 coincides with $(2)$.

Usually, 1-convex fuzzy processes are simply called convex fuzzy processes (see [7], [9]).

Example 1 Let us consider the fuzzy process $F:(0, \infty) \rightarrow \mathcal{F}(\mathbb{R})$ that associates to each $x \in(0, \infty)$ the points of the real line "much bigger than $\sqrt{x}$ ". Now, we define the fuzzy processes $F_{1}, F_{2}:(0, \infty) \rightarrow \mathcal{F}(\mathbb{R})$ as follows

$$
\begin{gathered}
F_{1}(x)(t)= \begin{cases}\frac{t}{\sqrt{x}}-1 & \text { if } \sqrt{x} \leq t \leq 2 \sqrt{x}, \\
1 & \text { if } t \geq 2 \sqrt{x}, \\
0 & \text { if } t \leq \sqrt{x},\end{cases} \\
F_{2}(x)(t)= \begin{cases}-\left(\frac{t-2 \sqrt{x}}{\sqrt{x}}\right)+1 & \text { if } \sqrt{x} \leq t \leq 2 \sqrt{x}, \\
1 & \text { if } t \geq 2 \sqrt{x} \\
0 & \text { if } t \leq \sqrt{x} .\end{cases}
\end{gathered}
$$

For $F_{1}$ and $x=4$, we have that the points of the real line "much bigger than $\sqrt{4}=2 "$ is the fuzzy set

$$
F_{1}(4)(t)= \begin{cases}\frac{t}{2}-1 & \text { if } 2 \leq t \leq 4, \\ 1 & \text { if } t \geq 4, \\ 0 & \text { if } t \leq 2,\end{cases}
$$

this means that the points after 4 are "much bigger than 2 ", while the points in the interval] 2, 4[ are partially "much bigger than 2 ", i.e., they have a degree of membership to the fuzzy set $F_{1}(4)$. Similarly, we can see that $F_{2}(4)$ also models 
the fuzzy set of the points of the real line "much bigger than 2 ". Therefore, both $F_{1}$ and $F_{2}$ model the fuzzy process $F$. Thus, we can find diverse fuzzy process that define $F$. Note that $F_{1}$ is $\frac{1}{2}$-convex, but $F_{2}$ is not s-convex for all $s \in(0,1]$.

\section{S-concave fuzzy process}

In this Section we introduce the concept of s-concave fuzzy process and we establish some properties. This concept generalizes the definition of concave set-valued function given in [4].

Definition 2 Let $s \in(0,1]$. A fuzzy process $F: C \subseteq \mathbb{R}^{m} \rightarrow \mathcal{F}\left(\mathbb{R}^{n}\right)$ is said to be a s-concave fuzzy process on $C$, if for all $a \in(0,1)$ and for all $x, y \in \mathbb{R}^{m}$ it satisfies the condition

$$
F\{(1-a) x+a y\} \subseteq(1-a)^{s} F(x)+a^{s} F(y) .
$$

1-concave fuzzy processes will be simply called concave fuzzy processes.

Example 2 Let us consider the fuzzy process $F:[0, \infty) \rightarrow \mathcal{F}(\mathbb{R})$, where $F(x)$ is the isosceles triangular fuzzy set with support $[-f(x), f(x)]$ where $f$ : $[0, \infty) \rightarrow \mathbb{R}$ is a s-convex function. It is easy to see that $F$ is s-concave.

Next we give a characterization for s-concave fuzzy processes by using the membership.

Theorem 1 Let $F: C \subseteq \mathbb{R}^{m} \rightarrow \mathcal{F}\left(\mathbb{R}^{n}\right)$ be a fuzzy process on $C$. Then, $F$ is s-concave if and only if

$$
F\left((1-a) x_{1}+a x_{2}\right)(y) \leq \sup _{y_{1}, y_{2}:(1-a)^{s} y_{1}+a^{s} y_{2}=y} \min \left\{F\left(x_{1}\right)\left(y_{1}\right), F\left(x_{2}\right)\left(y_{2}\right)\right\},
$$

for all $a \in(0,1)$ and for all $x, y \in C$.

Proof The result follows from Definition 2 and the addition and scalar multiplication on $\mathcal{F}\left(\mathbb{R}^{n}\right)$.

Now, we present another characterization by using the concept of support function of a fuzzy set. 
Theorem 2 Let $F: C \subseteq \mathbb{R}^{m} \rightarrow \mathcal{F}_{C}\left(\mathbb{R}^{n}\right)$ be a fuzzy process on $C$. Then, $F$ is s-concave if and only if $S(F(\cdot),(\alpha, \psi))$ is a s-convex function, that is, if and only if $S(F(\cdot),(\alpha, \psi))$ satisfies (1) for all $(\alpha, \psi) \in[0,1] \times \mathbb{S}^{m}$.

Proof Suppose that $F$ is a s-concave fuzzy process. Let $(\alpha, \psi) \in[0,1] \times \mathbb{S}^{m}$, $x_{1}, x_{2} \in \mathbb{R}^{n}$ and $a \in(0,1)$. Then, from the properties of the support function, we have that

$$
\begin{aligned}
S\left(F\left(a x_{1}+(1-a) x_{2}\right),(\alpha, \psi)\right) & \leq S\left(a^{s} F\left(x_{1}\right)+(1-a)^{s} F\left(x_{2}\right),(\alpha . \psi)\right) \\
& =\sigma\left(a^{s} F_{\alpha}\left(x_{1}\right)+(1-a)^{s} F_{\alpha}\left(x_{2}\right), \psi\right) \\
& =a^{s} \sigma\left(F_{\alpha}\left(x_{1}\right), \psi\right)+(1-a)^{s} \sigma\left(F_{\alpha}\left(x_{2}\right), \psi\right) .
\end{aligned}
$$

Consequently,

$S\left(F\left(a x_{1}+(1-a) x_{2}\right),(\alpha, \psi)\right) \leq a^{s} S\left(F\left(x_{1}\right),(\alpha, \psi)\right)+(1-a)^{s} S\left(F\left(x_{2}\right),(\alpha, \psi)\right)$.

Therefore, $S(F(\cdot),(\alpha, \psi))$ is s-convex. To prove the converse it suffices to show that

$$
S\left(F\left(a x_{1}+(1-a) x_{2}\right),(\alpha, \psi)\right) \leq S\left(a^{s} F\left(x_{1}\right)+(1-a)^{s} F\left(x_{2}\right),(\alpha, \psi)\right)
$$

for all $(\alpha, \psi) \in[0,1] \times \mathbb{S}^{m}$, which is a consequence of the properties of the support function of a fuzzy set.

Example 3 Let us consider the fuzzy process $F:[0, \infty) \rightarrow \mathcal{F}_{C}(\mathbb{R})$ given by

$$
F(x)(t)=\left\{\begin{array}{l}
\frac{t}{x^{s}} \text { si } 0 \leq t \leq x^{s}, \\
0 \quad \text { si } t \notin\left[0, x^{s}\right],
\end{array}\right.
$$

for $x \neq 0$ and $F(0)=\chi_{\{0\}}$. We have that the fuzzy support function $S(F(\cdot)$, $(\alpha, \psi))$, for each $(\alpha, \psi) \in[0,1] \times S^{1}$, with $S^{1}=\{-1,1\}$, is given by $S(F(x)$, $(\alpha, 1))=\alpha x^{s}$, which is a s-convex function and $S(F(x),(\alpha,-1))=0$ which is also s-convex. Then, from Theorem 2 we have that $F$ is a s-concave fuzzy process.

Proposition 1 Let $F: C \subseteq \mathbb{R}^{m} \rightarrow \mathcal{F}\left(\mathbb{R}^{n}\right)$ be a fuzzy process on $C$ such that

(a) $F(x+y) \subseteq F(x)+F(y)$,

(b) $F(t x)=t^{s} F(x)$.

Then $F$ is a s-concave fuzzy process on $C$.

Proof ¿From the addition and scalar multiplication on $\mathcal{F}\left(\mathbb{R}^{n}\right)$, and from conditions $(a)$ and $(b)$, we have that 


$$
\begin{aligned}
& F\left(a x_{1}+(1-a) x_{2}\right)(y) \\
\leq & \left(F\left(a x_{1}\right)+F\left((1-a) x_{2}\right)\right)(y) \\
= & \sup _{y_{1}, y_{2}: y_{1}+y_{2}=y} \min \left\{F\left(a x_{1}\right)\left(y_{1}\right), F\left((1-a) x_{2}\right)\left(y_{2}\right)\right\} \\
= & \sup _{y_{1}, y_{2}: a^{s} y_{1}+(1-a)^{s} y_{2}=y} \min \left\{F\left(a x_{1}\right)\left(a^{s} y_{1}\right), F\left((1-a) x_{2}\right)\left((1-a)^{s} y_{2}\right)\right\} \\
= & \sup _{y_{1}, y_{2}: a^{s} y_{1}+(1-a)^{s} y_{2}=y} \min \left\{\left(a^{s} F\left(x_{1}\right)\right)\left(a^{s} y_{1}\right),\left((1-a)^{s} F\left(x_{2}\right)\right)\left((1-a)^{s} y_{2}\right)\right\} \\
= & \sup _{y_{1}, y_{2}: a^{s} y_{1}+(1-a)^{s} y_{2}=y} \min \left\{F\left(x_{1}\right)\left(y_{1}\right), F\left(x_{2}\right)\left(y_{2}\right)\right\},
\end{aligned}
$$

for all $x_{1}, x_{2} \in C, a \in(0,1)$ and $y \in \mathbb{R}^{n}$. Therefore, by Theorem $1, F$ is a s-concave fuzzy process on $C$.

Example 4 Let $F: \mathbb{R}^{m} \rightarrow \mathcal{F}\left(\mathbb{R}^{n}\right)$ be a fuzzy quasilinear operator (see [10]), then $F$ satisfies the conditions in Proposition 1 for $s=1$. Thus, every fuzzy quasilinear operator is a concave fuzzy process.

\section{$5 \quad$ Hadamard's Inequality}

In this Section, we present some inequalities of Hadamard type for s-convex and s-concave fuzzy processes and we give some examples. With this aim, we first recall some basic concepts and properties of fuzzy random variables. A set-valued function $F:[0, b] \rightarrow \mathcal{K}\left(\mathbb{R}^{n}\right)$ is called Borel measurable, if its graph, i.e., the set $\{(t, x) / x \in F(t)\}$, is a Borel subset of $[0, b] \times \mathbb{R}^{n}$. Because the Lebesgue measure is complete, the Borel measurability of the set-valued mapping $F$ is equivalent to the following condition: for every Borel set $B \subseteq \mathbb{R}^{n}$, $F^{-1}(B)=\{t \in[0, b] / F(t) \cap B \neq \emptyset\} \in \mathbb{L}$, where $\mathbb{L}$ denotes the $\sigma$-algebra of all Lebesgue-measurable subsets of interval $[0, b]$. We will say that $F$ is measurable if $F$ is Borel measurable. Also, a measurable set-valued function $F:[0, b] \rightarrow \mathcal{K}\left(\mathbb{R}^{n}\right)$ is called a random set.

The integral of a measurable set-valued function $F:[0, b] \rightarrow \mathcal{K}\left(\mathbb{R}^{n}\right)$ is defined by

$$
\int_{0}^{b} F d t=\left\{\int_{0}^{b} f(t) d t / f \in S(F)\right\}
$$

where $\int_{0}^{b} f(t) d t$ is the Bochner-integral and $S(F)$ is the set of all integrable selectors of $F$, i.e.,

$$
S(F)=\left\{f \in L^{1}\left([0, b], \mathbb{R}^{n}\right) / f(t) \in F(t) \text { a.e. }\right\} .
$$


This definition was introduced by Aumann [11] as a natural generalization of the integration of single-valued functions.

A measurable set-valued function $F:[0, b] \rightarrow \mathcal{K}\left(\mathbb{R}^{n}\right)$ is said to be integrably bounded, if there exists a single-valued integrable function $h:[0, b] \rightarrow \mathbb{R}^{n}$ such that $\|x\| \leq h(t)$ for all $x$ and $t$ such that $x \in F(t)$.

If $F:[0, b] \rightarrow \mathcal{K}\left(\mathbb{R}^{n}\right)$ is an integrably bounded random set, then the Aumann integral of $F$ is a nonempty subset of $\mathbb{R}^{n}$.

If $\lambda \in \mathbb{R}$ and $F, F_{1}, F_{2}:[0, b] \rightarrow \mathcal{K}_{C}\left(\mathbb{R}^{n}\right)$ are integrably bounded random set, then

a) $\int_{0}^{b} F d t \in K_{C}\left(\mathbb{R}^{n}\right)$

b) $\int_{0}^{b}\left(\lambda F_{1}+F_{2}\right) d t=\lambda \int_{0}^{b} F_{1} d t+\int_{0}^{b} F_{2} d t$.

For details see Hiai and Umegaki [12].

Let $F:[0, b] \rightarrow \mathcal{F}_{K}\left(\mathbb{R}^{n}\right)$ be a fuzzy process and define $F_{\alpha}:[0, b] \rightarrow \mathcal{K}\left(\mathbb{R}^{n}\right)$ by $F_{\alpha}(x)=[F(x)]^{\alpha}, \forall \alpha \in[0,1]$. Then $F$ is called measurable if $F_{\alpha}$ is measurable for all $\alpha \in[0,1]$. Also, $F$ is called integrably bounded if $F_{\alpha}$ is an integrably bounded set-valued function for every $\alpha \in[0,1]$. If $F$ is a measurable fuzzy process, then $F$ is called a fuzzy random variable (f.r.v.) (see [13]).

Proposition 2 (Puri and Ralescu [13]) If $F:[0, b] \rightarrow \mathcal{F}_{K}\left(\mathbb{R}^{n}\right)$ is an integrably bounded f.r.v., then there exists a unique fuzzy set $u \in \mathcal{F}_{K}\left(\mathbb{R}^{n}\right)$ such that $[u]^{\alpha}=\int_{0}^{b} F_{\alpha} d t \forall \alpha \in[0,1]$.

The element $u \in \mathcal{F}_{K}\left(\mathbb{R}^{n}\right)$ in Proposition 2 defines the integral of the fuzzy random variable $F$ by $\int_{0}^{b} F d t=u \Leftrightarrow[u]^{\alpha}=\int_{0}^{b} F_{\alpha} d t$, for every $\alpha \in[0,1]$.

Theorem 3 If $F_{1}, F_{2}:[0, b] \rightarrow \mathcal{F}_{C}\left(\mathbb{R}^{n}\right)$ are integrably bounded f.r.v. and $\lambda \in \mathbb{R}$, then

$$
\int_{0}^{b}\left(\lambda F_{1}+F_{2}\right) d t=\lambda \int_{0}^{b} F_{1} d t+\int_{0}^{b} F_{2} d t .
$$

For more details and properties about the integral of f.r.v. see [13].

If $f:[a, b] \rightarrow \mathbb{R}$ is a convex function, then following inequalities hold,

$$
f\left(\frac{a+b}{2}\right) \leq \frac{1}{b-a} \int_{a}^{b} f(x) d x \leq \frac{f(a)+f(b)}{2}
$$

These inequalities are known in the literature as Hadamard's inequalities. Next we extend them. We first prove an inequality of Hadamard type for a s-convex 
fuzzy process and afterwards for s-concave fuzzy process.

Theorem 4 Let $F$ be a s-convex integrably bounded fuzzy process on an interval $I \subseteq[0, \infty)$ and let $a, b \in I$, with $a<b$. Then

$$
(s+1)^{-1}\{F(a)+F(b)\} \subseteq \int_{a}^{b} F(x) d x /(b-a) \subseteq 2^{s-1} F\left(\frac{a+b}{2}\right) .
$$

Proof Since $F$ is s-convex on $I$ we have that

$$
t^{s} F(a)+(1-t)^{s} F(b) \subseteq F\{t a+(1-t) b\}
$$

for all $t \in[0,1]$. Integrating this relation we get

$$
\begin{aligned}
\int_{0}^{1} F\{t a+(1-t) b\} d t & \supseteq \int_{0}^{1}\left\{t^{s} F(a)+(1-t)^{s} F(b)\right\} d t \\
& =F(a) \int_{0}^{1} t^{s} d t+F(b) \int_{0}^{1}(1-t)^{s} d t \\
& =(s+1)^{-1}\{F(a)+F(b)\} .
\end{aligned}
$$

Now, making the change of variable $x=t b+(1-t) a$, it follows the first relation in (4).

To prove the second relation in (4), observe that for all $x, y \in I$ we have that

$$
F\left(\frac{x+y}{2}\right) \supseteq \frac{1}{2^{s}}\{F(x)+F(y)\}
$$

Then taking $x=t a+(1-t) b$ and $y=t b+(1-t) a$, from (5) we obtain

$$
F\left(\frac{a+b}{2}\right) \supseteq \frac{1}{2^{s}}[F\{t a+(1-t) b\}+F\{t b+(1-t) a\}] .
$$

Integrating this relation we get

$$
\begin{aligned}
\int_{0}^{1} F\left(\frac{a+b}{2}\right) d t & \supseteq \int_{0}^{1} \frac{1}{2^{s}}[F\{t a+(1-t) b\}+F\{t b+(1-t) a\}] d t \\
& =\frac{1}{2^{s}}\left[\int_{0}^{1} F\{t a+(1-t) b\} d t+\int_{0}^{1} F\{t b+(1-t) a\} d t\right] .
\end{aligned}
$$


Since

$$
\int_{0}^{1} F\{t a+(1-t) b\} d t=\int_{0}^{1} F\{t b+(1-t) a\} d t=\frac{1}{b-a} \int_{a}^{b} F(x) d x
$$

it follows that

$$
\int_{a}^{b} F(x) d x /(b-a) \subseteq 2^{s-1} F\left(\frac{a+b}{2}\right) .
$$

Theorem 5 Let $F$ be a s-concave integrably bounded fuzzy process on an interval $I \subseteq[0, \infty)$ and let $a, b \in I$, with $a<b$. Then

$$
2^{s-1} F\left(\frac{a+b}{2}\right) \subseteq \int_{a}^{b} F(x) d x /(b-a) \subseteq(s+1)^{-1}\{F(a)+F(b)\} .
$$

Proof The proof is analogous to that of Theorem 4 .

Corollary 1 Let $F$ be a s-concave integrably bounded fuzzy process on an interval $I \subseteq[0, \infty)$ and let $a, b \in I$, with $a<b$. Then

$$
2^{s-1} \Gamma\left(\frac{a+b}{2}\right) \leq \int_{a}^{b} \Gamma(x) d x /(b-a) \leq(s+1)^{-1}(\Gamma(a)+\Gamma(b)),
$$

where $\Gamma=S(F(\cdot),(\alpha, \psi))$.

Proof The result follows from Theorem 5 and the properties of the fuzzy support function.

Example 5 Let us consider $s=1 / 2$ and the $1 / 2$-concave fuzzy process $F$ : $(1 / 2,1) \rightarrow \mathcal{F}(\mathbb{R})$ as in Example 3. Then

$$
\Gamma(x)=S(F(x),(\alpha, 1))=\alpha \sqrt{x}
$$

for each $\alpha \in[0,1]$. Thus, by Corollary 1 we have

$$
\frac{\sqrt{6}}{8} \alpha \leq \int_{1 / 2}^{1} \Gamma(x) d x \leq \frac{2+\sqrt{2}}{3} \alpha
$$




\subsection{Applications}

(a) For an integrably bounded fuzzy process $F:[0, b] \rightarrow \mathcal{F}_{C}\left(\mathbb{R}^{n}\right)$, the fuzzy integral mean of $F$ is a fuzzy process $M_{F}:(0, b] \rightarrow \mathcal{F}\left(\mathbb{R}^{n}\right)$ defined by

$$
M_{F}(x)=\frac{1}{x} \int_{0}^{x} F(t) d t, \forall x \in(0, b] .
$$

This concept was introduced in [9], where some properties are also studied. In [1] is studied the s-convexity of the fuzzy integral mean. The following Proposition gives a new relationship for the fuzzy integral mean, which is obtained by using the Hadamard inequality in Theorem 4.

Proposition 3 Let $F:[0, b] \rightarrow \mathcal{F}_{C}\left(\mathbb{R}^{n}\right)$ be a measurable integrably bounded fuzzy process. If $F$ is s-convex then $M_{F}$ is s-convex and

$$
(s+1)^{-1}\{F(0)+F(x)\} \subseteq M_{F}(x) \subseteq 2^{s-1} F\left(\frac{x}{2}\right) .
$$

Proof As $F$ is s-convex, then from Theorem 4.5 in [1] $M_{F}$ is s-convex. The relation (7) is an immediate consequence of Theorem 4 .

(b) With the aim of establishing some refinements of (3), Dragomir [14] introduced the mapping

$$
H(t)=\frac{1}{b-a} \int_{a}^{b} f\left(t x+(1-t) \frac{a+b}{2}\right) d x,
$$

and showed that if $f:[a, b] \rightarrow \mathbb{R}$ is a convex function, then $H(t)$ is convex and that

$$
f\left(\frac{a+b}{2}\right) \leq H(t) \leq \frac{1}{b-a} \int_{a}^{b} f(x) d x, \quad \forall t \in[0,1]
$$

Next, we extend this results for s-convex bounded fuzzy processes. Let $F$ : $[a, b] \rightarrow \mathcal{F}_{C}\left(\mathbb{R}^{n}\right)$ be an integrably bounded fuzzy process and define

$$
H_{F}(t)=\frac{1}{b-a} \int_{a}^{b} F\{t x+(1-t)(a+b) / 2\} d x,
$$


for $t \in[0,1]$.

Theorem 6 Let $F$ be a s-convex integrably bounded fuzzy process on an interval $[a, b]$. Then $H_{F}$ is s-convex on $[0,1]$ and

$$
H_{F}(t) \subseteq 2^{s-1} F\left(\frac{a+b}{2}\right), \quad \forall t \in[0,1] .
$$

Proof Let $t_{1}, t_{2} \in[0,1]$ and $\alpha, \beta \geq 0$ with $\alpha+\beta=1$. Then

$$
\begin{aligned}
& H_{F}\left(\alpha t_{1}+\beta t_{2}\right) \\
= & \frac{1}{b-a} \int_{a}^{b} F\left[\left(\alpha t_{1}+\beta t_{2}\right) x+\left\{1-\left(\alpha t_{1}+\beta t_{2}\right)\right\}(a+b) / 2\right] d x \\
= & \frac{1}{b-a} \int_{a}^{b} F\left[\alpha\left\{t_{1} x+\left(1-t_{1}\right)(a+b) / 2\right\}+\beta\left\{t_{2} x+\left(1-\beta t_{2}\right)(a+b) / 2\right\}\right] d x \\
\supseteq & \frac{1}{b-a} \int_{a}^{b} \alpha^{s} F\left\{t_{1} x+\left(1-t_{1}\right)(a+b) / 2\right\} d x+ \\
& \frac{1}{b-a} \int_{a}^{b} \beta^{s} F\left\{t_{2} x+\left(1-\beta t_{2}\right)(a+b) / 2\right\} d x \\
= & \alpha^{s} H_{F}\left(t_{1}\right)+\beta^{s} H_{F}\left(t_{2}\right),
\end{aligned}
$$

which shows that $H_{F}$ is s-convex. Now, let $t \in(0,1]$. Taking $r=t x+(1-$ $t)(a+b) / 2$ we obtain

$$
H_{F}(t)=\int_{q}^{p} F(r) d r /(p-q)
$$

where $p=t b+(1-t)(a+b) / 2$ and $q=t a+(1-t)(a+b) / 2$. By Theorem 4 we have that

$$
\int_{q}^{p} F(r) d r /(p-q) \subseteq 2^{s-1} F\left(\frac{p+q}{2}\right)=2^{s-1} F\left(\frac{a+b}{2}\right),
$$

what proves $(8)$.

Remark 1 Proceeding as in the proof of Theorem 6, it can be also shown that if $F$ is a s-concave integrably bounded fuzzy process on an interval $[a, b]$, then

$$
2^{s-1} F\left(\frac{a+b}{2}\right) \subseteq H_{F}(t)
$$




\section{Jensen's Inequality}

In this Section we give a generalization of the Jensen inequality for s-convex and s-concave fuzzy processes.

Theorem 7 Let $F: C \subseteq \mathbb{R}^{m} \rightarrow \mathcal{F}\left(\mathbb{R}^{n}\right)$ be a s-convex fuzzy process on $C$ and $s>0$. Then we have the relation

$$
\sum_{i=1}^{n} p_{i}^{s} F\left(x_{i}\right) \subseteq F\left(\sum_{i=1}^{n} p_{i} x_{i}\right)
$$

whenever $p_{i} \geq 0, x_{i} \in C$ and $\sum_{i=1}^{n} p_{i}=1$. If $F$ is a s-concave fuzzy process on $C$ and $s>0$. Then we have the relation

$$
F\left(\sum_{i=1}^{n} p_{i} x_{i}\right) \subseteq \sum_{i=1}^{n} p_{i}^{s} F\left(x_{i}\right)
$$

whenever $p_{i} \geq 0, x_{i} \in C$ and $\sum_{i=1}^{n} p_{i}=1$.

Proof We first show (9). To do this, we proceed by induction on $n$. For $n=2$, (9) is the definition of s-convexity of $F$. Now, suppose that (9) holds for $n=k-1$ and given $p_{i} \geq 0, x_{i} \in C$ and $\sum_{i=1}^{k} p_{i}=1$, we may and do assume that all $p_{i}>0$. Let $q_{j}=p_{j} /\left(p_{1}+\ldots+p_{k-1}\right), 1 \leq j<k$. Then $q_{1}+\ldots+q_{k-1}=1$ and thus

$$
q_{1}^{s} F\left(x_{1}\right)+\ldots+q_{k-1}^{s} F\left(x_{k-1}\right) \subseteq F\left(q_{1} x_{1}+\ldots+q_{k-1} x_{k-1}\right) .
$$

Put $P=p_{1}+\ldots+p_{k-1}$, then

$$
\begin{aligned}
F\left(p_{1} x_{1}+\ldots+p_{k} x_{k}\right) & =F\left\{P\left(\frac{p_{1}}{P} x_{1}+\ldots+\frac{p_{k-1}}{P} x_{k-1}\right)+p_{k} x_{k}\right\} \\
& \supseteq P^{s} F\left(\frac{p_{1}}{P} x_{1}+\ldots+\frac{p_{k-1}}{P} x_{k-1}\right)+p_{k}^{s} F\left(x_{k}\right) \\
& \supseteq P^{s}\left(\frac{p_{1}^{s}}{P^{s}} F\left(x_{1}\right)+\ldots+\frac{p_{k-1}^{s}}{P^{s}} F\left(x_{k-1}\right)\right)+p_{k}^{s} F\left(x_{k}\right) \\
& =\sum p_{i}^{s} F\left(x_{i}\right)
\end{aligned}
$$

which establishes (9) for $n=k$, and hence for all $n \in \mathbb{N}$. The proof of (10) follows the same steps and so we omit it. 
Corollary 2 Let $F: C \subseteq \mathbb{R}^{m} \rightarrow \mathcal{F}\left(\mathbb{R}^{n}\right)$ a s-convex fuzzy process on $C$ and $s>0$. Then

$$
n^{-s} \sum_{i=1}^{n} F\left(x_{i}\right) \subseteq F\left(n^{-1} \sum_{i=1}^{n} x_{i}\right)
$$

whenever $x_{i} \in C, 1 \leq i \leq n$.

Example 6 We consider the 1/2-convex fuzzy process $F_{1}$ from Example 1. Thus, from Corollary 2, for each $\alpha \in[0,1]$ we have that

$$
\left[(1+\alpha) \sqrt{n^{-1} \sum_{i=1}^{n} x_{i}}, \infty\right) \supseteq n^{-1 / 2}(1+\alpha) \sum_{i=1}^{n}\left[\sqrt{x_{i}}, \infty\right) .
$$

\section{References}

[1] Y. Chalco-Cano, M.A. Rojas-Medar and R. Osuna-Gómez, S-Convex fuzzy processes, Computer and Mathematics with Applications, in press, (2003).

[2] W.W. Breckner, Stetigkeitsaussagen für eine Klasse verallgemeinerter konvexer Funtionen in topologischen linearen Räumen, Publ. Inst. Math. (Beograd.), 23, 13-20, (1978).

[3] H. Hudzik and L. Maligranda, Some remarks on s-convex functions, Aequationes Mathematicae, 48, 100-111, (1994).

[4] W.W. Breckner, Continuity of generalized convex and generalized concave setvalued functions, Rev Anal. Numér. Théor. Approx., 22, 39-51, (1993).

[5] R. Lowen, Convex fuzzy sets, Fuzzy Sets and Systems, 3, 291-310, (1980).

[6] M.A. Rojas-Medar, R.C. Bassanezi and H. Román-Flores, A generalization of the Minkowski embedding theorem and applications, Fuzzy Sets and Systems, 102, 263-269, (1999).

[7] Yu-Ru, Chin-Yao Low and Tai-Hsi Wu, A note on convex fuzzy processes, Applied Mathematics Letters, 15, 193-196 (2002).

[8] M. Matloka, Convex fuzzy processes, Fuzzy Sets and Systems, 110, 104-114, (2000).

[9] Y. Chalco-Cano, M.A. Rojas-Medar and H. Román-Flores, M-Convex fuzzy mapping and fuzzy integral mean, Computers and Mathematics with Applications, 40, 1117-1126, (2000).

[10] M.D. Jiménez-Gamero, Y. Chalco-Cano, M.A. Rojas-Medar and A.J.V. Brandão, Fuzzy quasilinear spaces ad applications, submitted to publication. 
[11] R.J. Aumann, Integral of set-valued functions, J. Math. Anal. Appl., 12, 1-11, (1965).

[12] F. Hiai and H. Umegaki, Integrals, conditional expectations, and martingales of multivalued functions, J. Multivar. Anal., 7, 149-182, (1977).

[13] M.L. Puri and D.A. Ralescu, Fuzzy random variables, J. Math. Anal. Appl., 114, 301-317, (1987).

[14] S.S. Dragomir, Two Mappings in Connection to Hadamard's Inequalities, $J$. Math. Anal. Appl., 167, 49-56 (1992). 